Institute of Neurology, University of Bari G Defazio

V Lepore

D Acquistapace

P Livrea

Department of

Neurology, University of Rome

A Berardelli

Institute of Neurology, University of Genova

C Abbruzzese

Department of

Preventive Medicine

ASL BA1, Andria

V Coviello

Institute of Neurology, University of Trieste

L Capus

Istituto Nazionale Neurologico C Besta, Milan

F Carella

Institute of

Ophthalmology, University of Naples

M T De Berardinis

Institute of Neurology, S Raffaele Hospital,

Milan

G Galardi

2nd Neurologic Clinic, University of Messina

P Girlanda

2nd Neurologic Clinic, University of Firenze S Maurri

Institute of Neurology, "Università Cattolica del Sacro Cuore",

Rome

A Albanese

Institute of Neurology, University of Verona

L Bertolasi

Institute of Neurology, University of Bologna R Liguori

Institute of Neurology, University of Perugia A Rossi

2nd Neurologic Clinic, University of Naples

L Santoro

"Consorzio Mario Negri Sud", S Maria Imbaro, Italy

G Tognoni

Correspondence to: Dr Giovanni Defazio, Institute of Neurology of the University of Bari, Piazza Giulio Cesare 11, I-70124 Bari, Italy. Telephone 0039 80 5478511; fax 003980 5478532 .

Received 20 March 1997 and in revised form 13 June 1997 Accepted 30 June 1997

\title{
Possible risk factors for primary adult onset dystonia: a case-control investigation by the Italian Movement Disorders Study Group
}

\author{
Giovanni Defazio, Alfredo Berardelli, Giovanni Abbruzzese, Vito Lepore, \\ Vincenzo Coviello, Diomira Acquistapace, Livio Capus, Francesco Carella, \\ Maria Teresa De Berardinis, Giuseppe Galardi, Paolo Girlanda, Silvio Maurri, \\ Alberto Albanese, Laura Bertolasi, Rocco Liguori, Aroldo Rossi, Lucio Santoro, \\ Gianni Tognoni, Paolo Livrea
}

\begin{abstract}
Objectives-Little is known about the aetiology of idiopathic adult onset dystonia. The Italian Movement Disorders Study Group promoted a case-control study on some hypothetical risk factors including past medical events, life events, life habits, occupational hazards, and family hystory of dystonia, parkinsonism, and tremor.

Methods-Cases affected by idiopathic adult onset dystonia (age at symptom onset $>20$ years, duration of disease $>$ one year and $<$ five years) were selected among consecutive outpatients attending 14 Italian centres. Control outpatients matched for age ( \pm 5 years), sex, and referral centre were identified among diagnostic categories thought to be unassociated with study exposures. Information was obtained by a standardised questionnaire administered by medical interviewers. Conditional logistic univariate and multivariate regression analyses were performed by a standard statistical package.
\end{abstract}

Results-Multivariate analysis on 202 cases and 202 age and sex matched control outpatients indicated that head or facial trauma with loss of consciousness, family history of dystonia, and family history of postural tremor independently increased the risk of developing adult onset dystonia, whereas hypertension and cigarette smoking exerted a protective effect. The findings also suggested a positive association between local body injury-for example, previous ocular diseases and neck or trunk trauma-and dystonia of the same body part.

Conclusions-The results support the idea that environmental and genetic factors may both be important in the aetiology of adult onset dystonia, and suggest aetiological clues worthy of further analytical investigation.

(F Neurol Neurosurg Psychiatry 1998;64:25-32)

Keywords: adult onset dystonia; trauma; eye diseases; cigarette smoking; family history of dystonia; family history of tremor
Little is known about the aetiology of primary adult onset dystonia. Evidence of familial aggregation $^{12}$ seems to argue in favour of heredity, and segregation analysis ${ }^{3}$ has suggested an autosomal dominant transmission and reduced penetrance or, alternatively, polygenic inheritance. Neurophysiological findings ${ }^{56}$ point to an individual predisposition triggered by external factors. ${ }^{12} 7-11$

To our knowledge, no published data are available on the distribution of potentially important risk factors among patients with adult onset dystonia and matched controls. The lack of information prompted us to conduct an exploratory case-control study, pooling cases from several clinical centres throughout Italy. This provided a large sample size and allowed us to screen numerous factors for possible association with adult onset dystonia.

\section{Methods}

CASE PATIENTS

Cases were selected from January to July 1995 among consecutive outpatients attending for follow up visits at the movement disorder clinics of one ophthalmological and 13 neurological Institutions belonging to the Italian Movement Disorders Study Group. Six centres were in northern Italy, four in central Italy, and four in southern Italy. The "Consorzio Mario Negri Sud" (S Maria Imbaro, Italy) provided methodological advice. Inclusion criteria were as follows: a diagnosis of focal, segmental, or multifocal dystonia (with or without dystonia associated tremor) according to published standard criteria $^{12}$; age at onset of dystonia (defined as time of first symptom) >20 years; and duration of disease >one year (to minimise possible misclassification error in the aetiological diagnosis) and < five years (to reduce recall bias). Exclusion criteria were: neurological abnormalities in addition to dystonia except tremor associated with dystonia ${ }^{13}$; a history of exposure to dopamine receptor blocking agents within six months before the onset of dystonia; "severe" head trauma (loss of consciousness for more than six hours, or major surgical procedures, or both); features suggesting dopa responsive dystonia, paroxysmal dystonia, and alcohol responsive myoclonic dystonia as well as other secondary dystonias ${ }^{14}$; and features suggesting ophthalmological, orthopaedic, and psychogenic causes of dystonia. ${ }^{15-17}$ 
CONTROLS

For each case we recruited one control outpatient matched for age ( +5 years), sex, and referral centre. Controls were identified among outpatients with idiopathic hemifacial spasm (undergoing botulinum toxin therapy, with unilateral sciatica undergoing EMG examination, and with non-neoplastic gastroenterological diseases undergoing sonography or endoscopy. Duration of disease was never higher than five years. Potential controls were excluded if they had received treatment with drugs known to induce extrapyramidal side effects, or they were related to a case involved in the study. The first suitable control admitted to the clinic after case inclusion was recruited.

Data collection and definition of variables was obtained by a standardised questionnaire administered face to face by 14 medical interviewers (one for each centre). To maximise reliability between and within interviewers they received training before the study. Case patients were questioned about the period preceding the onset of adult onset dystonia; control patients about the same pre-exposure period selected for their matched case patients.

Table 1 Distibution of risk factors potentially responsible for brain lesions in case patients with $(n=122)$ and without $(n=80)$ head CT or MRI

\begin{tabular}{llcl} 
& \multicolumn{2}{l}{ Head imaging studies } & \\
\cline { 2 - 3 } Risk factor & Yes (\%) & No (\%) & p Value \\
\hline Diabetes & 4.9 & 5 & NS \\
Hypertension & 14.7 & 17.5 & NS \\
Thyroid diseases & 4.1 & 3.7 & NS \\
General anaesthesia & 29.5 & 16.2 & $<0.05$ \\
Other diseases & 23.8 & 22.5 & NS \\
Cigarette smoking (current+past smokers) & 37 & 36 & NS \\
Wine drinking (current+past drinkers) & 41.7 & 42.5 & NS \\
Head trauma & 13.9 & 0 & $<0.01$ \\
$\quad$ With loss of consciousness & 6.5 & 5 & NS \\
Without loss of consciousness & & & \\
\hline
\end{tabular}

Table 2 Demographic features and distribution of risk factors in control outpatients according to the diagnostic category

\begin{tabular}{|c|c|c|c|}
\hline & $\begin{array}{l}\text { Hemifacial } \\
\text { spasm }\end{array}$ & Sciatalgia & $\begin{array}{l}\text { Gastroenterological } \\
\text { disease }\end{array}$ \\
\hline No $(M / F)$ & $106 \quad(32 / 74)$ & $51 \quad(20 / 31)$ & $45 \quad(21 / 24)$ \\
\hline Mean age (SD) & $57.4(9.6)$ & $52.7(11.3$ & $52.2(15.9)$ \\
\hline Mean disease duration (SD) & $3.0(1.1)$ & $2.7(0.9)$ & $3.2(1.4)$ \\
\hline Education level $>5$ th grade & 24 & 10 & 11 \\
\hline Marital status (married-cohabitant) & 78 & 36 & 34 \\
\hline Life events & 29 & 19 & 15 \\
\hline Cigarette smoking (current smokers) & 28 & 19 & 15 \\
\hline Wine drinking (current drinkers) & 39 & 23 & 19 \\
\hline Manual work & 38 & 19 & 15 \\
\hline Pesticides & 5 & 3 & 1 \\
\hline Factory toxic substances & 11 & 4 & 3 \\
\hline \multicolumn{4}{|l|}{ Head or face trauma without loss of } \\
\hline $\begin{array}{l}\text { Head or face trauma with loss of } \\
\text { consciousness }\end{array}$ & 3 & 1 & 1 \\
\hline Neck or trunk trauma & 2 & 0 & 1 \\
\hline Arm trauma & 7 & 5 & 4 \\
\hline Leg trauma & 3 & 3 & 2 \\
\hline Diabetes & 5 & 3 & 3 \\
\hline Hypertension & $36^{\star}$ & 7 & 7 \\
\hline Thyroid diseases & 4 & 3 & 1 \\
\hline Eye diseases & 7 & 6 & 5 \\
\hline Anxiety & 24 & 7 & 8 \\
\hline Depression & 11 & 4 & 4 \\
\hline Other diseases & 20 & 12 & 8 \\
\hline General anaesthesia & 25 & 11 & 10 \\
\hline \multicolumn{4}{|l|}{ Family history of: } \\
\hline Tremor & 2 & 0 & 1 \\
\hline Parkinsonism & 4 & 0 & 2 \\
\hline Dystonia & 0 & 1 & 0 \\
\hline
\end{tabular}

${ }^{\star} \mathrm{p}<0.05$; significantly different from the other diagnostic categories.
The questionnaire collected data on age, sex, age at symptom onset, educational level (assessed as years of schooling), and marital status; previous medical and surgical events including diabetes, hypertension, thyroid and ocular diseases, anxiety, depression, other diseases (for example, autoimmune and endocrine diseases), general anaesthesia, non-severe head or facial trauma (with or without loss of consciousness), neck or trunk trauma (without injury to the head or loss of consciousnes) severe enough to require medical attention but no major surgery, and limb trauma; life events, including severe disease or death of a person very close to the patient, separation and divorce, migration inside or outside of Italy, loss of job, severe financial problems; work, occupational exposure to pesticides or factory processes involving chemicals, toxic fumes, or materials and poisons, wine drinking and cigarette smoking; and family history (first degree relatives only) of postural non-parkinsonian and non-alcohol related tremor, parkinsonism, and dystonia. The time interval between risk factor and onset of symptoms was recorded.

Information on medical history was supported by medical records or detailed reports of specific treatments. First degree relatives were considered to be affected by dystonia, parkinsonism, or postural tremor only if at least one other family member provided adequate information (probable diagnosis), or one of the investigators personally saw the secondary case (definite diagnosis). Diagnosis of essential tremor was made according to published criteria. ${ }^{18}$ Questions on cigarette smoking and wine drinking referred to the year preceding onset of symptoms: subjects were classified as never having smoked (non-smokers) or drank (non-drinkers), past and current smokers, or drinkers.

\section{DATA ANALYSIS}

Exposure variables were represented in the model by a single indicator variable $(1$, if the subject was exposed; 0, if not). A standard statistical package (EGRET) was used for a conditional logistic univariate regression analysis (taking into account the matching on age, sex, and clinical centre), and odds ratios (ORs), two sided $95 \%$ confidence intervals (95\% CIs), and $\mathrm{p}$ values (likelihood ratio statistic) were calculated. p Values $<0.05$ were considered to be significant. Case-control pairs containing missing information were excluded from the analysis. To check for type II error, the statistical power relative to each variable was assessed by the equation for matched case-control study reported by Schlesselman. ${ }^{19}$ Multivariate analysis was performed according to the model building strategy proposed by Hosmer and Lemeshaw. ${ }^{20}$ Because of the large number of variables for the number of observations, we used the 0.5 level at the univariate test as a screening criterion for selecting candidate variables. ${ }^{20}$ After fitting the model containing all the selected variables, we deleted the unimportant variables and fitted a new model. The importance of variables was assessed by examining the significance of each $p$ value and 
Table 3 Conditional logistic univariate regression analysis relative to the variables that failed to reach significance with primary adult onset dystonia

\begin{tabular}{|c|c|c|c|c|c|c|c|}
\hline Risk factor & OR $(95 \% C I)$ & $p$ Value & $+/+$ & $-/+$ & $+/-$ & $-1-$ & $?$ \\
\hline Marital status (married-cohabitant) & $0.95(0.59$ to 1.52$)$ & 0.82 & 116 & 32 & 34 & 20 & 0 \\
\hline Life events & $1.48(0.93$ to 2.37$)$ & 0.09 & 34 & 29 & 43 & 96 & 0 \\
\hline Cigarette smoking (current smokers) & $0.66(0.41$ to 1.05$)$ & 0.08 & 18 & 44 & 28 & 112 & 0 \\
\hline Wine drinking (current + past drinkers) & $0.78(0.51$ to 1.20$)$ & 0.27 & 47 & 47 & 37 & 71 & 0 \\
\hline Wine drinking (current drinkers) & $0.74(0.52$ to 1.21$)$ & 0.27 & 39 & 42 & 30 & 91 & 0 \\
\hline Manual work & $1.59(0.99$ to 2.52$)$ & 0.06 & 43 & 29 & 45 & 85 & 0 \\
\hline Pesticides & $0.67(0.23$ to 1.87$)$ & 0.44 & 0 & 9 & 6 & 186 & 1 \\
\hline Factory toxic substances & $1.07(0.52$ to 2.20$)$ & 0.85 & 4 & 14 & 15 & 168 & 1 \\
\hline $\begin{array}{l}\text { Head or face trauma without loss of } \\
\text { consciousness }\end{array}$ & $1.35(0.51$ to 3.73$)$ & 0.50 & 0 & 9 & 12 & 181 & 0 \\
\hline Arm trauma & $0.42(0.14$ to 1.10$)$ & 0.08 & 0 & 16 & 8 & 178 & 0 \\
\hline Leg trauma & $1.60(0.55$ to 4.98$)$ & 0.46 & 1 & 7 & 10 & 184 & 0 \\
\hline Diabetes & $0.91(0.39$ to 2.14$)$ & 0.83 & 0 & 11 & 10 & 181 & 0 \\
\hline Thyroid diseases & $1.00(0.35$ to 2.85$)$ & 1.00 & 1 & 7 & 7 & 186 & 1 \\
\hline Anxiety & $1.26(0.78$ to 2.02$)$ & 0.34 & 7 & 31 & 39 & 124 & 1 \\
\hline Depression & $1.92(0.98$ to 3.76$)$ & 0.06 & 6 & 13 & 25 & 158 & 0 \\
\hline Other diseases & $1.24(0.76$ to 2.02$)$ & 0.38 & 11 & 29 & 36 & 126 & 0 \\
\hline General anaesthesia & $1.09(0.68$ to 1.73$)$ & 0.70 & 12 & 34 & 37 & 119 & 0 \\
\hline Family history of parkinsonism & $1.5(0.53$ to 4.20$)$ & 0.44 & 0 & 6 & 10 & 186 & 0 \\
\hline
\end{tabular}

comparing the estimated ORs from the new and the old models. This process of deleting, refitting, and verifying continued until it yielded a model containing the essential variables only (main effects model). Given the imperfect match on age, estimates were adjusted for this variable. For variables significantly associated with adult onset dystonia in multivariate modelling, we evaluated, among the case patients, the temporal sequence (or direction of the association) by testing (Pearson's $\chi^{2}$ test) the association between the exposure and duration of disease measured by symptom onset and categorised in two levels: $>$ two years and $\leqslant$ two years. Thereafter, we considered the need for including biologically plausible multiplicative interaction terms among the variables. ${ }^{19}{ }^{20}$ Finally, goodness of fit - the appropriateness of the logistic model in describing the observed data-was assessed by calculating the estimated logistic probabilities for individual case-control pairs, the changes in the variance of the logistic model after deleting poorly fit pairs, and the values of $\delta \beta$ for each pair. ${ }^{2021}$

\section{Results}

Two hundred and ten case patients met eligibility criteria, but eight refused to take part to the study. Thus, 202 case patients ( 73 men and 129 women aged 20 to 70 years) were analysed. Mean age at symptom onset was 52.1 (SD 11.6) years and mean disease duration was 3.1 (SD 1.3) years. Focal dystonia was found in 158 cases $(78 \%)$ : there were 90 cases with blepharospasm, 44 with cervical dystonia , 23 with arm dystonia, and one with leg dystonia. Segmental or multifocal dystonia was found in 44 case patients: there were 32 cases with blepharospasm, 22 with oromandibular dystonia, 15 with cervical dystonia, five with laryngeal dystonia, two with arm dystonia, three with trunk dystonia, and one with leg dystonia. In 13 cases with cervical dystonia there was dystonia associated tremor. Six patients had dystonia at more than two sites.

Head CT, or MRI, or both disclosed no abnormality in 122 cases; in the other 80 cases $(40 \%)$ no imaging studies were available. Head CT, or MRI, or both were available for all case patients who had a history of head trauma with loss of consciousness. Scans (MRI) excluding spinal cord lesions were also available for all cases with neck or trunk trauma. Case patients without neuroradiological studies did not have a significantly higher frequency of risk factors for brain damage than cases with normal head imaging (table 1). Cases without head imaging studies had a significantly longer duration of disease than those with studies (3.4 (SD 1.3) years $v 2.8(\mathrm{SD} 1.4)$ years, $\mathrm{p}=0.006)$.

Stratifying the cases by duration of disease showed no increase in the relative frequency of risk factors across strata as the duration of disease increased (not shown).

There were 202 control outpatients. Refusal rate was $<3 \%$. Table 2 shows the demographic and clinical features and distribution of risk factors among diagnostic categories. The proportion of patients reporting hypertension was significantly higher in the idiopathic hemifacial spasm group than in the other diagnostic groups.

\section{UNIVARIATE ANALYSIS}

Table 3 shows the crude ORs relative to the variables which failed to reach significance. Smoking status was analysed by collapsing past and never smokers. Taking non-smokers as reference yielded ORs of $0.66(95 \% \mathrm{Cl} 0.40$ to $1.07)$ for current smokers and of $0.98(95 \% \mathrm{Cl}$ 0.52 to 1.87 ) for past smokers. In past smokers, the interval elapsing between stopping smoking and the onset of dystonia ranged from three to 15 years. Current smokers had a significantly lower age at onset of dystonia (mean, 46.8 (SD 12.8 ) years) than either past smokers (mean, 52.6 (SD 12.9) years) or never smokers (mean, 53.9 (SD 11.1) years) (one way analysis of variance (ANOVA)): $F=6.01, \quad \mathrm{p}=0.003$; Newman-Keuls' test: “current smokers" different from the other groups, $\mathrm{p}<0.05$ ).

The study had an estimated $<80 \%$ chance of detecting threefold modification in the risk of developing dystonia with $\alpha=0.05$ (two sided) for diabetes, thyroid diseases, head or facial trauma without loss of consciousness, and limb trauma. The other variables calculated yielded a study power $>90 \%$. 
Table 4 Conditional logistic univariate regression analysis relative to the variables that reached significance with primary adult onset dystonia

\begin{tabular}{|c|c|c|c|c|c|c|c|}
\hline Risk factor & OR $(95 \% C I)$ & $p$ Value & $+/+$ & $-/+$ & $+/-$ & $-/-$ & $?$ \\
\hline Educational level ( $>5$ th grade) & $0.59(0.37$ to 0.97$)$ & 0.04 & 9 & 36 & 17 & 140 & 0 \\
\hline Hypertension & $0.50(0.28$ to 0.88$)$ & 0.02 & 14 & 36 & 18 & 134 & 0 \\
\hline Head or face trauma with loss of consciousness & $4 \quad(1.12$ to 14.3$)$ & 0.03 & 0 & 5 & 17 & 180 & 0 \\
\hline Neck or trunk trauma & $5.55(1.20$ to 25.0$)$ & 0.03 & 1 & 2 & 11 & 188 & 0 \\
\hline Eye diseases & $3.3(1.63$ to 6.67$)$ & 0.002 & 7 & 11 & 34 & 151 & 0 \\
\hline Family history of tremor & 8.5 (2.64 to 45.9$)$ & $<0.001$ & 1 & 2 & 23 & 176 & 0 \\
\hline Family history of dystonia & $16 \quad(2.4$ to 682$)$ & $<0.001$ & 0 & 1 & 15 & 186 & 0 \\
\hline
\end{tabular}

$+/+=$ case and control exposed; $-/+=$ control only; $+/-=$ case only; $-/-=$ neither exposed; ? = case-control pairs containing missing information.

Table 4 shows the crude ORs relative to the variables that reached significance. The variable "education level" was dichotomised according to demographic and statistical considerations. About half of case patients $(n=112)$ had an education level $\leqslant 5$ th grade (the first Italian education stage); the remaining 90 case patients were equally distributed among the next three education stages.

Questionnaire data showed that $7.3 \%$ of the case patients with cranial dystonia and $7.5 \%$ of the case patients with extracranial dystonia had previous head or face trauma with loss of consciousness (lasting some minutes to two hours). Neck or trunk trauma was reported by $12 \%$ of case patients with cervical dystonia $v$ $3.5 \%$ of cases with other types of dystonia $(z=1.97, \mathrm{p}=0.049)$. The intervals elapsing between head or face and neck or trunk trauma and onset of adult onset dystonia were 1-10 (mean 4.7) years and 1-7 (mean 4.2) years respectively.

Patients with blepharospasm had a higher frequency of eye diseases than patients without $(25.4 \%$ v $10 \% ; z=2.53, \mathrm{p}=0.01)$. In patients with blepharospasm $(n=122)$, prior blepharitis and keratoconjunctivitis (14.7\%) were significantly more frequent $(\mathrm{p}<0.01)$ than prior glaucoma $(2.5 \%)$, cataract $(3.3 \%)$, and retinopathy $(4.1 \%)$. In patients with dystonia other than blepharospasm, prior eye diseases were equally distributed. The interval elapsing between eye diseases and the onset of dystonia ranged from one to five years (mean 3.2).

Questionnaires from $7.4 \%$ of case patients reported a family history of dystonia; most affected relatives had the same type of dystonia as the cases. The proportion of affected persons was significantly higher $(\mathrm{p}=0.002)$ in case relatives $(15 / 1374=0.011)$ than in control relatives $(1 / 1246=0.0008)$; the latter proportion was similar to the estimated prevalence of adult onset dystonia in the general population $(295 / 1000000=0.0003) .{ }^{22}$ Twenty four case patients $(12 \%)$ reported a family history of postural tremor. Two case patients with cervical dystonia had family members who had

Table 5 Results of conditional logistic multivariate regression analysis (the final model was fitted on 202 matched sets)

\begin{tabular}{lcc}
\hline Risk factor & OR (95\% CI) & p Value \\
\hline Hypertension & $0.36(0.16$ to 0.65$)$ & 0.002 \\
Eye diseases & $3.11(1.38$ to 7.02$)$ & 0.006 \\
Head or face trauma with loss of consciousness & $3.2(1.04$ to 10.1$)$ & 0.042 \\
Neck or trunk trauma & $7.84(1.35$ to 45.7$)$ & 0.022 \\
Cigarette smoking (current smokers) & $0.51(0.29$ to 0.89$)$ & 0.018 \\
Family history of dystonia & $10.9(1.39$ to 84.9$)$ & 0.023 \\
Family history of tremor & $5.66(1.81$ to 17.7$)$ & 0.003 \\
\hline
\end{tabular}

tremor in the same body part-the neck. The others had more diffuse tremor. Three case patients reported family history of both dystonia and tremor (in different family members). Ten case patients had relatives with a definite diagnosis of dystonia, and 14 case patients and two control patients had relatives with a definite diagnosis of essential tremor. Limiting the analysis to the definite diagnosis yielded significant associations for dystonia $(95 \% \mathrm{Cl}$ 2.86 to infinite; $\chi^{2}$ with continuity correction=8.1, $\mathrm{p}=0.004)$ and tremor $(\mathrm{OR}=7.45$; $95 \% \mathrm{Cl} 1.67$ to $68.1 ; \mathrm{p}=0.003)$.

MULTIVARIATE ANALYSIS

The main effects model (table 5) included eye diseases, head or face trauma with loss of consciousness, neck or trunk trauma, and family history of tremor and dystonia as independent risk factors, and hypertension and smoking as independent protective factors for adult onset dystonia. No relation was found between the above variables and duration of dystonia categorised in two levels: > two years and $\leqslant$ two years. We tested the possibility of entering into the model the biologically plausible interactions among the variables. Interaction terms including family history of dystonia could not be calculated, however, because few casecontrol pairs showed the combined risk factors needed for calculating interaction. Other interaction terms never attained $\mathrm{p}$ values $\leqslant 0.2$. Assessing the goodness of fit enabled us to identify only three pairs that had small estimated probabilities (two of them had also a large $\delta \beta$ value) and induced major changes $(>4)$ in the deviance of the logistic model if they were removed from the data set. We did not exclude these pairs from the analysis because they were biologically plausible and had no effect on the selection of variables during the various steps of model building.

To verify whether eye diseases related more strongly to blepharospasm than to other dystonias, we fitted the above model with more homogeneous case groups-that is, the 122 patients with blepharospasm (with or without other dystonias), and the 80 cases with dystonia other than blepharospasm. This yielded a significant association of eye diseases with the outcome in the blepharospasm group $(\mathrm{OR}=4.5$, $95 \%$ CI 1.6 to $12.5, \mathrm{p}=0.003$ ) but not in the non-bletharospasm group $(\mathrm{OR}=1.2,95 \% \mathrm{CI}$ 0.24 to $5.6, p=0.85$ ). Analysis of the other variables yielded significant ORs for hypertension (bletharospasm group: $\mathrm{OR}=0.3,95 \%$ CI 0.13 to $0.7, \mathrm{p}=0.004$; non-bletharospasm group: $\mathrm{OR}$ 
$0.24,95 \%$ CI 0.07 to $0.86, p=0.03$ ) and family history of tremor (bletharospasm group: $\mathrm{OR}=9.3,95 \% \mathrm{CI} 1.9$ to $46.1, \mathrm{p}=0.007$; nonblepharospasm group: OR 6.3, 95\% CI 1.24 to $32.2, p=0.026)$ in both groups. Although the ORs calculated for head or face trauma with loss of consciousness (bletharospasm group: $\mathrm{OR}=3.0,95 \%$ CI 0.6 to $14, \mathrm{p}=0.19$; nonbletharospasm group: OR 3.5, 95\% CI 0.7 to 18, $\mathrm{p}=0.13$ ) and smoking (bletharospasm group: $\mathrm{OR}=0.46,95 \% \mathrm{CI} 0.2$ to $1.1, \mathrm{p}=0.07$; non-bletharospasm group: OR $0.55,95 \% \mathrm{CI}$ 0.27 to $1.15, \mathrm{p}=0.11$ ) did not reach significance, the $95 \%$ CIs made a positive association with both cranial and extracranial dystonia likely. Family history of dystonia could be calculated in neither group because few casecontrol pairs showed the combination of risk factor necessary for analysis. Neither could neck or trunk trauma be estimated in the blepharospasm group, whereas a significant association was found in the other group ( $\mathrm{OR}=6.7,95 \%$ CI 1.03 to $43 ; \mathrm{p}=0.046)$. Restricting multivariate analysis to the 59 patients with cervical dystonia yielded a higher adjusted estimate for neck or trunk trauma $(\mathrm{OR}=11.2,95 \%$ CI 1.3 to $95, \mathrm{p}=0.03)$.

\section{Discussion}

The case-control design is a powerful and costeffective strategy to generate testable hypotheses on the aetiology of diseases with low incidence and prevalence. Thus we performed an exploratory case-control study investigating the association between primary adult onset dystonia and several potential risk factors. The results indicated that head or face trauma with loss of consciousness and family history of dystonia as well as of tremor independently increased the risk of developing adult onset dystonia, whereas hypertension and cigarette smoking exerted a protective effect. In addition, we found a positive association between local injury to a body part, prior ocular diseases and neck or trunk trauma, and dystonia of that body part.

Because the study was not population based, we cannot rule out a bias in patient selection. To correct for this we not only included a large number of clinics in various areas of Italy but during the study period we recruited $96 \%$ of the consecutive outpatients who met eligibility criteria. Apart from the large number of patients with focal dystonia, our case population had demographic and clinical features similar to those of previous clinical series. ${ }^{12}$ Differences in methods used for selecting patients, including the criteria for duration of disease, may explain the higher frequence of patients with focal dystonia in our series. Although $40 \%$ of our case patients had no CT or MRI of the head, eligibility criteria made contamination from secondary dystonia unlikely. As others have underlined, when neither the history nor the report of neurological signs mentions incongruities other than those directly attributable to dystonia, adult onset dystonia is rarely due to an identifiable pathology. ${ }^{23}$ In addition, we found no significant difference in the frequency of risk factors potentially responsible for brain damage in patients with and those without imaging studies.

Although we did not base the study on newly diagnosed cases, the relatively short average duration of disease reduced, without eliminating, the potential for recall bias. Using prevalent cases may be a potential source of survival bias, ${ }^{19}$ but the low incidence of adult onset dystonia in the general population ${ }^{22}$ made it unfeasible to use incident cases. None the less, survival bias seems unlikely because stratifying the cases by duration of disease showed no increase in the relative frequency of risk factors across strata as disease duration lengthened.

As in any case-control study, there is the possibility that cases and controls are not drawn from the same demographic groups or are not equally motivated to provide accurate anamnestic data. To minimise these possibilities, we used control outpatients, who may be more motivated to search their memories than healthy controls. Furthermore, controls were matched to case patients by age and sex, and attended outpatient centres of the same hospital. These were large academic hospitals providing both primary care and complex neurological and non-neurological services. Thus the referral network that brought cases and controls to our institutions was probably the same. We took control outpatients from three diagnostic categories thought to be unassociated with study exposures. ${ }^{24} 25$ Supporting this assumption, control groups had comparable exposure rates for most risk factors under study. The fact that more subjects in the idiopathic hemifacial spasm group than in the other diagnostic groups had hypertension may have produced an artificial difference between cases and controls, thus making the protective association between hypertension and adult onset dystonia less secure. Yet when we excluded the variable "hypertension" from the model the significance of the other estimates remained unchanged.

Multivariate analysis failed to confirm several potential risk factors, including life events, manual work, occupational hazards, wine drinking, head or face trauma without loss of consciousness, limb trauma, antecedent diabetes, thyroid diseases, anxiety, depression, other diseases, general anaesthesia, and family history of parkinsonism. Most of these negative findings were probably valid and accurate because differential recall between cases and controls was unlikely for most exposures; in addition, all variables except diabetes, thyroid diseases, head or face trauma without loss of consciousness, and limb trauma achieved satisfactory study power. The lack of association between a history of psychiatric illnesses and adult onset dystonia contributes to the large body of evidence refuting the once commonly held view that psychological factors are important in the aetiology of this disorder.

Multivariate analysis indicated a significant association between adult onset dystonia and head or face trauma with loss of consciousness. Estimation of trauma in the subgroups with 
cranial and extracranial dystonia failed to reach significance (probably owing to a loss of statistical power). Yet $95 \%$ CIs in both groups made a positive association likely. It is possible that patients with adult onset dystonia may have better recall for head or face trauma than controls because of their own "lay belief" as to what caused their disease. If so, controls with head associated symptoms (idiopathic hemifacial spasm) and those without (sciatica and gastroenterological symptoms) should have different recall for head or face trauma. But they did not. Events inducing a loss of consciousness should be recalled equally well by case and control patients. Hence the association between head or face trauma with loss of consciousness and adult onset dystonia may have aetiological relevance. It must be stressed that the case patients with loss of consciousness of more than six hours were excluded from the study, but this criterion was not applied to controls. Thus the significant $\mathrm{OR}$ for head or face trauma may be underestimated.

Previous studies have suggested that trauma inducing basal ganglia or brainstem lesions can cause focal or segmental dystonia. ${ }^{7}{ }^{10}$ Yet our patients had neither clinical nor radiographical evidence of brain damage. The suggested role of peripheral trauma in inducing topographically related dystonia ${ }^{710}{ }^{11}$ should be considered, even if the area involved makes it difficult or impossible to exclude direct discrete brain damage. In favour of a discrete central damage was the comparable frequency of head or face trauma with loss of consciousness in patients with cranial or extracranial dystonia. Among other trauma, the positive association between neck or trunk trauma and dystonia outside the cranial area-namely cervical dystonia-in patients without evidence of spinal cord lesions adds to the growing body of uncontrolled data linking local injury to topographical related dystonia. ${ }^{71011}$

The positive association between eye diseases and blepharospasm ${ }^{1}$ may also be in line with the above idea. Or it might merely reflect an information bias. Patients with blepharospasm may of course be more likely than patients with extrapalpebral dystonia or controls to see an ophthalmologist and hence to have a diagnosis of eye disease. If so, eye diseases should have been more frequent in idiopathic hemifacial spasm than in other controls, yet they were not. Among the eye diseases, blepharitis and keratoconjunctivitis were more frequent than cataract, glaucoma, and retinopathy in patients in blepharospasm; whereas they were equally distributed in patients with dystonia other than blepharospasm. Blepharitis and keratoconjunctivitis may intensify the trigeminopalpebral reflexes, which are known to be altered in patients with cranial dystonia. ${ }^{5}$

An intrinsic limitation in case-control design is the difficulty in firmly establishing the temporal sequence between exposure and disease. Because onset of dystonia may be prolonged and difficult to detect, ${ }^{26}$ trauma in the cranial-cervical area and eye diseases may follow dystonia (cause and effect bias) and, therefore, would not influence risk. Despite this limitation, two points lead us to think that the observed associations are not secondary to dystonia: (1) no association emerged between any of the above variables and duration of adult onset dystonia; (2) more than a year elapsed between eye diseases or trauma and the onset of dystonia. These long time intervals do not necessarily argue against a causal relation because delayed onset dystonia has been reported after injuries. ${ }^{72} 28$ Many variables, including severity, location, timing, and type of initial injury probably contribute to the delay in onset of dystonia. ${ }^{728}$

The negative association between smoking and adult onset dystonia may be an artifact of selective mortality or the consequence of confounding by other factors. To our knowledge, there are no data to support the selective mortality of patients with adult onset dystonia who smoke. Multivariate analysis also excluded a potentially confounding role of several variables. Obviously, the list of conceivable confounders is almost inexhaustible. For example, although we did not examine the effect of coffee drinking, smokers are more likely than non-smokers to drink coffee. Another possibilty is a cause and effect bias. Supporting this view we failed to detect any significant effect of past smoking. On the other hand, our cases stopped smoking three to 15 years before the onset of adult onset dystonia and some data indicate that the risk for many smoking associated diseases can decrease substantially within some years of stopping smoking. ${ }^{29}$ Patients with blepharospasm might also have avoided smoking because it aggravates their symptoms, perhaps even before the eye condition has been diagnosed. Yet the subgroup of patients with blepharospasm and those with dystonia other than blepharospasm had similar ORs. Alternatively, smoking might have a protective effect. This is supported by the strength of the association (roughly a halving of risk) and the evidence of a similar finding in another extrapyramidal disorder, Parkinson's disease. ${ }^{30}$ Under this hypothesis, it would be expected that adult onset dystonia would have a later onset in smokers than in non-smokers. But in our sample it did not. Smokers in whom adult onset dystonia developed reported an earlier onset of disease than past smokers and patients who had never smoked. This "paradoxical age at onset effect" may be a further argument suggesting a spurious association between smoking and adult onset dystonia. Interestingly, a similar age at onset effect has been found in Parkinson's disease, ${ }^{30}$ linked in this setting to bias in detemining the age at onset. Overall, the potential association between smoking and adult onset dystonia deserves further investigation, given the potential implications for our understanding of the mechanisms underlying adult onset dystonia.

In accordance with studies indicating a genetic contribution to primary adult onset dystonia, $^{34}$ a significantly larger number of case patients reported first degree relatives affected by dystonia. This result might reflect the influence of various biases. Firstly, the 
reason why we found a lower rate of familial occurrence of dystonia than did previous series $^{34}$ could be that we identified affected relatives from the case patients' answers to the questionnaire rather than by clinical examination of all at risk relatives. This bias probably tended to underestimate the effect. Secondly, an awareness or family information bias could have intervened because unlike controls, who tend to be unacquainted with the disease, cases may be more aware of other family members affected by dystonia. ${ }^{31}$ And lastly, by excluding controls related to case patients in the study we may have increased the probability of selecting controls without familial aggregation of dystonia. However, the proportion of affected relatives among controls was comparable with the estimated prevalence of adult onset dystonia in the general population. ${ }^{22}$ Hence our finding seems valid even though it probably underestimated the strength of the association. Nevertheless, ORs and their 95\% CIs indicate that familial aggregation of dystonia may increase the risk of developing adult onset dystonia more than the other variables investigated. A misclassification error in the diagnosis of adult onset dystonia must also be considered because data on family history were, at least partly, self reported. But we confirmed the association between family history of dystonia and adult onset dystonia by analysing the definite diagnoses only-that is, the secondary cases personally seen.

In agreement with previous uncontrolled studies suggesting a link between tremor, possibly essential tremor, and dystonia ${ }^{32} 33$ we found significantly more patients reporting first degree relatives affected by postural tremor (non-parkinsonian or due to alcohol). A family information bias seems unlikely to explain the familial clustering of postural tremor in our series. The potential misclassification error in distinguishing the aetiological and clinical type of tremor should also be considered, because postural tremor may be a feature of many neurological disorders. ${ }^{20}$ Yet, again, we detected a positive association between a family history of essential tremor and adult onset dystonia when we considered only the definite diagnoses. Possible explanations for this association may be the coexistence in the same pedigrees of both adult onset dystonia and essential tremor, ${ }^{32}$ or a genetic link between the two disorders. ${ }^{13}{ }^{34}$ Postural tremor may be also an initial manifestation of dystonia. ${ }^{13}$ Hence we cannot be sure that relatives reported as having tremor alone actually had dystonia. Genetic linkage studies may be ultimately needed to clarify the relation between adult onset dystonia and essential tremor.

In summary, our findings suggest that family history of dystonia or tremor and head or face trauma with loss of consciousness may be independently associated with an increasing risk of developing adult onset dystonia, whereas cigarette smoking may exert a protective effect. They also suggest a positive association between local body injury - for example, prior ocular diseases and neck or trunk trauma-and dystonia of the same body part.
The ORs and their 95\% CIs indicate that familial, possibly genetic, factors may have a role greater than the other investigated environmental factors. Although we tried to minimise sources of bias likely to distort the findings, the intrinsic limitations of the casecontrol design to investigate a wide range of variables might have influenced the soundness of inferences. Nevertheless, in this exploratory study we sought not to establish definitive protective and risk factors for adult onset dystonia, but to learn about possible causes of the disease and to identify aetiological clues worthy of further analytical investigation. A priority in designing future studies is to account adequately for the concurrent or interactive influence of genetic factors and for the potential aetiological heterogeneity of the various forms of adult onset dystonia.

GD thanks Mr Alberto Defazio for assistance during the preparation of the manuscript. Statistical support by Professor Giorgio Assennato is gratefully aknowledged.

\section{Appendix: List of participants}

G Laddomada, Institute of Neurology, University of Bari, Italy; P Marchetti, L Vacca, Department of Neurology, University of Rome, Italy; R Marchese, C Serrati, Institute of Neurology, University of Genova, Italy; $M$ Mucchiut, Institute of Neurology, University of Trieste, Italy; $\mathrm{V}$ Fetoni, Istituto Nazionale Neurologico C Besta, Milan, Italy; P Calace, G Cortese, Institute of Ophthalmology, University of Naples, Italy; L Maderna, Institute of Neurology, S Raffaele Hospital, Milan, Italy; A Trusso, 2nd Neurologic Clinic, University of Messina, Italy; S Brogelli, 2nd Neurologic Clinic, University of Firenze, Italy; AR Bentivoglio, A Romigi, the Institute of Neurology, "Università Cattolica del Sacro Cuore", Rome, Italy; C Cordivari, Institute of Neurology, University of Bologna, Italy; F Lalli, N Tambasco, Institute of Neurology, University of Perugia, Italy; C Crisci, F Crispi, 2nd Neurologic Clinic, University of Naples, Italy; F Carinci, the "Consorzio Mario Negri Sud", S Maria Imbaro, Italy.

1 Grandas F, Elston J, Quinn N, Marsden CD. Blepharospasm: a review of 264 patients. I Neurol Neurosurg Psychiatry $1988 ; 51: 767-72$.

2 Jankovic J, Ford J. Blepharospasm and orofacial-cervical dystonia: clinical and pharmacological findings in 100 patients. Ann Neurol 1988;13:402-11.

3 Waddy HM, Fletcher MD, Harding AE, Marsden CD. A genetic study of idiopathic focal dystonias. Ann Neurol 1991;29:320-24.

4 Defazio G, Livrea P, Guanti G, Lepore V, Ferrari E. Genetic contribution to idiopathic adult-onset blepharospasm and contribution to idiopathic adult-onset blepharospasm

5 Berardelli A, Rothwell JC, Day BL, Marsden CD. The pathophysiology of cranial dystonia. In: S Fahn, CD Marsden, DB Calne, eds. Advances in neurology. Vol 50. New York: Raven Press, 1988:525-35.

6 Pauletti G, Berardelli A, Cruccu G, Agostino R, Manfredi M. Blink reflex and the masseter inhibitory reflex in patients with dystonia. Mov Disord 1993;8:495-500.

7 Burton LS, Jankovic J. Delayed-onset progressive movement disorders after static brain lesions. Neurology 1996;46:6874.

8 Nutt JG. Meige syndrome and thyroid dysfunction. Neurology 1984;(suppl 1):222.

9 Klawans HL. Dystonia and tremor following exposure to 2,3,7,8,-tetrachlorodibenzo-p-dioxin. Mov Disord 1987;2: $2,3,7,8$,

10 Jankovic J. Post-traumatic movement disorders: central and peripheral mechanisms. Neurology 1994;44:2006-14. 
11 Fletcher NA, Harding AE, Marsden CD. The relationship between trauma and idiopathic torsion dystonia. 7 Neurol between trauma and idiopathic torsion
Neurosurg Psychiatry 1991;54:713-7.

12 Fahn S. Concept and classification of dystonia. In: S Fahn, CD Marsden, DB Calne, eds. Advances in neurology. Vol 50. New York: Raven Press, 1988:1-8.

13 Jedynak CP, Bonnett AM, Agid Y. Tremor and idiopathic dystonia. Mov Disord 1991;6:230-6.

14 Calne DB, Lang AE. Secondary dystonia. In: S Fahn, CD Marsden, DB Calne, eds. Advances in neurology. Vol 50. New York: Raven Press, 1988:9-33.

15 Salorio PD, Conte RQ. Ophthalmologic causes of blepharospasm. In: J Jankovic, $\mathrm{E}$ Tolosa, eds. Advances in neurology. Vol 49. New York: Raven Press, 1988:91-102.

16 Suchowersky O, Calne DB. Non dystonic causes of torticollis. In: S Fahn, CD Marsden, DB Calne, eds. Advances in neurology. Vol 50. New York: Raven Press, 1988:501-8.

17 Fahn S, Williams DT. Psychogenic dystonia. In: S Fahn, CD Marsden , DB Calne, eds. Advances in neurology. Vol 50. New York: Raven Press, 1988:431-55.

18 Hubble JP, Busenbark KL, Koller WC. Essential tremor. Clin Neuropharmacol 1989;12:453-82.

19 Schlesselmann JJ. Case-control studies. Oxford: Oxford Schlesselmann JJ. Cas
University Press, 1982.

20 Hosmer DW, Lemeshaw S. Applied logistic regression. New York: John Wiley, 1989

21 Hosmer DW, Taber S, Lemeshaw S. The importance of assessing the fit of logistic regression models: a case study. Am f Public Health 1991;81:1630-5.

22 Nutt JG, Muenter MD, Aronson A, Kurland LT, Melton LJ. Epidemiology of focal and generalized dystonia in Rochester, Minnesota. Mov Disord 1988;3:188-94.

23 Marsden CD. Investigation on dystonia. In: S Fahn, CD Marsden, DB Calne, eds. Advances in neurology. Vol 50. New York: Raven Press, 1988:35-43.
24 Wacholder S, Mclaughlin JK, Silverman DT, Mandel JS. Selection of controls in case-control studies. 1: Principles. Am f Epidemiol 1992;135:1019-28.

25 Wacholder S, Mclaughlin JK, Silverman DT, Mandel JS. Selection of controls in case-control studies. 2: Types of controls. Am f Epidem 1992;135:1029-41.

26 Micheli S, Fernandez-Pardal M, Quesada P, Brannan T, Obeso JA. Variable onset of adult inherited focal dystonia: a problem for genetic study. Mov Disord 1994;9:64-8.

27 Burke RE, Fahn S, Gold AP. Delayed onset dystonia in patients with static encephalopathy. $\mathcal{F}$ Neurol Neurosurg Psychiatry 1980;43:789-97.

28 Lee MS, Rinne JO, Ceballos-Baumann A, Thompson PD, Marsden CD. Dystonia after head trauma. Neurology 1994; 44:1374-8.

29 Kahn HA. The Dorn study of smoking and mortality among US veterans: report on eight and one half years of observation. In: Haenszel W, ed. Epidemiologic approaches to the study of cancer and other chronic diseases. National Cancer Institute monograph. Bethesda, MD: US Department of Health, Education and Welfare, 1966:19;1-25.

30 Morens DM, Grandinetti A, Reed D, White LR, Ross GW. Cigarette smoking and protection from Parkinson' disease: false association or etiologic clue? Neurology 1995;45: $1041-51$.

31 Sackett DL. Bias in analytical research. F Chron Dis 1979;32:51-63.

32 Jankovic J, Leder S, Warner D, Schwartz K. Cervical dystonia: clinical findings and associated movement disorders. Neurology 1991;41:1088-91.

33 Dubinsky RM, Gray CS, Koller WC. Essential tremor and dystonia. Neurology 1993;43:2382-4.

34 Lou JS, Jankovic J. Essential tremor: clinical correlates in 350 patients. Neurology 1991;41:234-8. 